\title{
DETERMINACIÓN DE LA MÁXIMA RETENCIÓN DE COMPUESTOS BIOACTIVOS Y CAPACIDAD ANTIOXIDANTE EN EL NÉCTAR DE TOMATE DE ÁRBOL (Solanum betaceaum Cav.)
}

\author{
Daniella S. Rojas Benites ${ }^{a^{*}}$, Ritva Repo de Carrasco $^{\mathrm{b}}$, Christian R. Encina Zelada $^{\mathrm{c}}$
}

\begin{abstract}
RESUMEN
Se establecieron los parámetros apropiados para elaborar néctar de tomate de árbol (Solanum betaceaum Cav.) con una mayor retención en el contenido de ácido ascórbico, compuestos fenólicos, carotenoides totales y capacidad antioxidante presentes en la materia prima. Se determinó el contenido de compuestos bioactivos en la pulpa de tomate de árbol: carotenoides totales con 4,27 mg $\beta$-caroteno/100g, vitamina $C$ con $28,83 \mathrm{mg}$ de ácido ascórbico/100g, compuestos fenólicos con 100,55 mg ácido gálico equivalente $/ 100 \mathrm{~g}$ y una capacidad antioxidante de 3,31 $\mu \mathrm{mol}$ trolox/g medida por el método DPPH y 4,65 $\mu$ mol trolox/g medida por el método ABTS. Se obtuvo una mayor retención de los compuestos bioactivos y capacidad antioxidante en el néctar de tomate de árbol con un $\mathrm{pH}$ de 3,33; $13^{\circ} \mathrm{Brix}$, dilución pulpa:agua 1:2,5 y una temperatura de pasteurización de $99,5^{\circ} \mathrm{C}$ por un minuto, encontrándose un contenido de carotenoides totales de $1,68 \mathrm{mg} \beta$-caroteno $/ 100 \mathrm{~g}$, vitamina $\mathrm{C}$ de $11,45 \mathrm{mg}$ de ácido ascórbico/100g, compuestos fenólicos de 32,96 mg ácido gálico equivalente $/ 100 \mathrm{~g}$ y capacidad antioxidante de $1,38 \mu \mathrm{mol}$ trolox/g medida por el método DPPH y 2,00 $\mu \mathrm{mol}$ trolox/g medida por el método ABTS.
\end{abstract}

Palabras clave: tomate de árbol, ácido ascórbico, compuestos fenólicos, carotenoides totales, capacidad antioxidante.

\section{DETERMINATION OF MAXIMUN RETENTION OF BIOACTIVE COMPOUNDS AND ANTIOXIDANT CAPACITY ON NECTAR OF TREE TOMATO (Solanum betaceaum Cav.)}

\footnotetext{
ABSTRACT

The appropriate parameters to produce nectar of tree tomato (Solanum betaceaum Cav.), with a greater retention on the content of ascorbic acid, phenolic compounds, total carotenoids

${ }^{a}$ Ingeniero en Industrias Alimentarias. Facultad de Industrias Alimentarias, Universidad Nacional Agraria La Molina, Avenida La Molina, Lima 15024, Perú, drojas@lamolina.edu.pe

b Profesor Principal del Departamento de Ingeniería de Alimentos, Facultad de Industrias Alimentarias, Universidad Nacional Agraria La Molina, Lima - Perú.

c Profesor Asociado del Departamento de Tecnología de Alimentos, Facultad de Industrias Alimentarias, Universidad Nacional Agraria La Molina, Lima - Perú.
} 
and antioxidant capacity that are present in the raw material, were established. The content of bioactive compounds in the tree tomato pulp was determined: total carotenoids with 4,27 $\mathrm{mg} \beta$-carotene $/ 100 \mathrm{~g}$, vitamin $\mathrm{C}$ with $28,83 \mathrm{mg}$ ascorbic acid/ $100 \mathrm{~g}$, phenolic compounds with $100,55 \mathrm{mg} \mathrm{AGE} / 100 \mathrm{~g}$ and the antioxidant capacity with 3,3172 $\mu \mathrm{mol}$ trolox/g measured by the DPPH method and 4,6551 $\mu \mathrm{mol}$ trolox/g measured by the ABTS method. A greater retention on the bioactive compounds and antioxidant capacity on the tree tomato nectar was obtained at a $\mathrm{pH}$ of $3,33,{ }^{\circ}$ Brix of 13 , dilution water:pulp of $1: 2,5$ and a pasteurization temperature of $99,5^{\circ} \mathrm{C}$ for 1 minute, finding on it a content of $1,68 \mathrm{mg} \beta$-carotene $/ 100 \mathrm{~g}$, vitamin $\mathrm{C}$ of $11,45 \mathrm{mg}$ de ascorbic acid $/ 100 \mathrm{~g}$, phenolic compounds of de $32,96 \mathrm{mg} \mathrm{AGE} / 100 \mathrm{~g}$ $\mathrm{y}$ the antioxidant capacity of de $1,3803 \mu \mathrm{mol}$ trolox/g measured by the DPPH method and $2,0006 \mu \mathrm{mol}$ trolox/g measured by the ABTS method.

Key words: tree tomato, ascorbic acid, phenolic compounds, total carotenoids, antioxidant capacity, nectar.

\section{INTRODUCCIÓN}

La importancia adquirida en los últimos años, en relación al consumo de frutas y vegetales, como alternativa para prevenir diversas enfermedades cardiovasculares, neurológicas y cancerígenas, atribuyéndole esta propiedad a la presencia de compuestos bioactivos en su composición, que poseen actividad antioxidante y contribuyen a la defensa del organismo ${ }^{1}$, permite desarrollar nuevas propuestas en el consumo de diversas variedades de frutas. Dentro del grupo de frutas nativas, de gran potencial agroindustrial procedentes de la sierra del Perú, se encuentra el tomate de árbol (Solanum betaceaum Cav.) perteneciente a la familia de las solanáceas, originaria de los Andes peruanos, dispersa en otros países de la región andina como Chile, Ecuador, Bolivia, Brasil y Colombia. Apreciado por sus excelentes cualidades nutritivas, sensoriales y su uso versátil; fuente de vitaminas A (1000 U.I), C (25 mg/ 100 g), E y complejo B (niacina, tiamina, riboflavina y piridoxina), bajo nivel en hidratos de carbono (menos de 40 calorías), rico en minerales, pectina y fibra que ayudan a prevenir el estreñimiento y a reducir la tasa de colesterol en la sangre ${ }^{15}$. A pesar de estas cualidades, el cultivo de tomate de árbol no ha logrado alcanzar un desarrollo a nivel industrial y comercial en el Perú. Por lo señalado anteriormente, la investigación se orientó a evaluar el efecto de los factores de $\mathrm{pH}$, ${ }^{\circ}$ Brix y dilución durante la estandarización y la temperatura de pasteurización en los compuestos bioactivos y capacidad antioxidante durante la elaboración de néctar de tomate de árbol (Solanum betaceaum Cav.) con la finalidad de obtener información sobre una industrialización adecuada que garantice la difusión de una bebida alimenticia con propiedades nutritivas y saludables favorables para el consumidor.

\section{PARTE EXPERIMENTAL}

\section{Lugar de ejecución}

La investigación se realizó en el Laboratorio de Biotecnología e Instrumentación, 
Laboratorio de Análisis Físico-Químico de los Alimentos, Laboratorio de Investigación y en la Planta Piloto de Tecnología de Alimentos de la Facultad de Industrias Alimentarias y en el Laboratorio de Análisis Químico del Departamento de Química de la Facultad de Ciencias, en las instalaciones de la Universidad Nacional Agraria La Molina.

\section{Materiales y reactivos}

Muestra: Se trabajó con tomate de árbol (Solanum betaceaum Cav.) variedad amarilla, procedente del distrito de Chinchao, Provincia de Huánuco, Departamento de Huánuco.

Reactivos: ABTS (2,2' azinobis (3-ethylbenoxthiazoline 6-sulfonate acid) de Sigma Aldrich Co. ${ }^{\circledR}$; DPPH (2,2 difenil-1-picrilhidrazil) de Sigma Aldrich Co. ${ }^{\circledR}$; etanol absoluto y ácido bórico de Merck; metanol, hexano , ácido sulfúrico, ácido clorhídrico, biftalato de potasio de J.T. Baker; folin-Ciocalteau $2 \mathrm{~N}$, trolox, ácido gálico, $\beta$ Caroteno, ácido ascórbico de Sigma Aldrich Co. ${ }^{\circledR}$; acetona, ácido acético glacial, hidróxido de sodio, cloruro de sodio y alcohol isopropílico de Fermont; sulfato de cobre pentahidratado (Sulfato cúprico), thiourea de Merck; 2,4 Dinitrofenilhidrazina (DNP), ácido metafosfórico y persulfato de potasio de Harleco; diclorometano de Riedel-de-Haë, y carbonato de sodio y bicarbonato de sodio de Mallinckrodt.

\section{Métodos de análisis}

- Análisis físicos ${ }^{13}$ : colorimétrico, diámetro y largo, peso, firmeza, rendimiento en pulpa.

- Análisis fisicoquímicos ${ }^{3}$ : humedad, proteína, grasa, fibra bruta, cenizas, carbohidratos, $\mathrm{pH}$; acidez titulable, sólidos solubles.

- Análisis químico ${ }^{\mathbf{2 , 4 5 9}}$ : ácido ascórbico, compuestos fenólicos totales, carotenoides totales, capacidad antioxidante.

- Análisis microbiológico: recuento total de aerobios viables, recuento total de hongos y levaduras.

\section{Metodología experimental \\ Caracterización de la materia prima}

Se realizó la caracterización de los frutos de tomate de árbol mediante los siguientes análisis: físicos (diámetro, largo, peso, color y firmeza), fisicoquímicos (proximal, $\mathrm{pH}$, sólidos solubles ( ${ }^{\mathrm{B}}$ Brix), acidez titulable).

Luego del escaldado, pulpeado y refinado de la materia prima, se evaluó el rendimiento y se procedió a realizar la determinación del contenido de compuestos bioactivos y capacidad antioxidante en la pulpa, antes de ser sometida al proceso de estandarización y tratamiento térmico. Finalmente, se realizó el análisis microbiológico de recuento total de aerobios viables y recuento total de mohos y levaduras en la pulpa. 
Determinación de los factores $(\mathbf{p}<\mathbf{0 , 0 1})$ que influyen en la retención del ácido ascórbico, compuestos fenólicos y capacidad antioxidante en la estandarización y tratamiento térmico en la elaboración del néctar de tomate de árbol.

Se determinaron los factores que influyen significativamente en la retención del ácido ascórbico, compuestos fenólicos y capacidad antioxidante durante la elaboración de néctar de tomate de árbol. Los factores de $\mathrm{pH},{ }^{\circ}$ Brix, dilución de pulpa y temperatura del tratamiento térmico con sus respectivos niveles se muestran en el cuadro 1 .

En la tabla 1 se presenta el flujo de operaciones para la elaboración del néctar de tomate de árbol.

Tabla 1. Factores y sus niveles considerados en el diseño experimental Taguchi $\mathrm{L}_{9}\left(3^{4}\right)$

\begin{tabular}{lccc}
\hline \multicolumn{1}{c}{ FACTORES } & MÁXIMO & MEDIO & MÍNIMO \\
\hline $\mathrm{F}_{1}:$ pH del néctar & 3,7 & 3,5 & 3,3 \\
$\mathrm{~F}_{2}:{ }^{\circ}$ Brix del néctar & 14 & 13 & 12 \\
$\mathrm{~F}_{3}$ : Dilución de pulpa: agua & $1: 4$ & $1: 3,5$ & $1: 3$ \\
$\mathrm{~F}_{4}:$ Temperatura del tratamiento térmico $\left({ }^{\circ} \mathrm{C}\right)$ & 95 & 90 & 85 \\
\hline
\end{tabular}

Determinación del nivel adecuado para los factores que influyen significativamente en la retención del ácido ascórbico, compuestos fenólicos y capacidad antioxidante en la estandarización y tratamiento térmico en la elaboración del néctar de tomate de árbol.

Para una mayor retención del contenido de ácido ascórbico, compuestos fenólicos y capacidad antioxidante en la elaboración del néctar de tomate de árbol se empleó el método de Superficie de Respuesta $(\alpha=0,05)$, juntamente con la función deseabilidad para un análisis multirespuesta, haciendo uso de las variables significativas que resultaron de la evaluación por el método Taguchi en la etapa anterior.

\section{Caracterización del producto final}

En base al resultado de las etapas de investigación, se elaboró el néctar de tomate de árbol con los factores que hacen máxima la retención ácido ascórbico, compuestos fenólicos y la capacidad antioxidante durante la elaboración. Se caracterizó el producto a través de las siguientes evaluaciones: análisis proximal, $\mathrm{pH}$, sólidos solubles ( $\left.{ }^{\circ} \mathrm{Brix}\right)$, acidez titulable, color, vitamina $\mathrm{C}$, carotenoides totales, compuestos fenólicos, capacidad antioxidante y evaluación sensorial. Para verificar la eficiencia del tratamiento térmico, así como las buenas prácticas de manufactura durante la elaboración, se realizaron los siguientes análisis microbiológicos: detección de microorganismos aerobios mesófilos viables y recuento total de hongos y levaduras. 


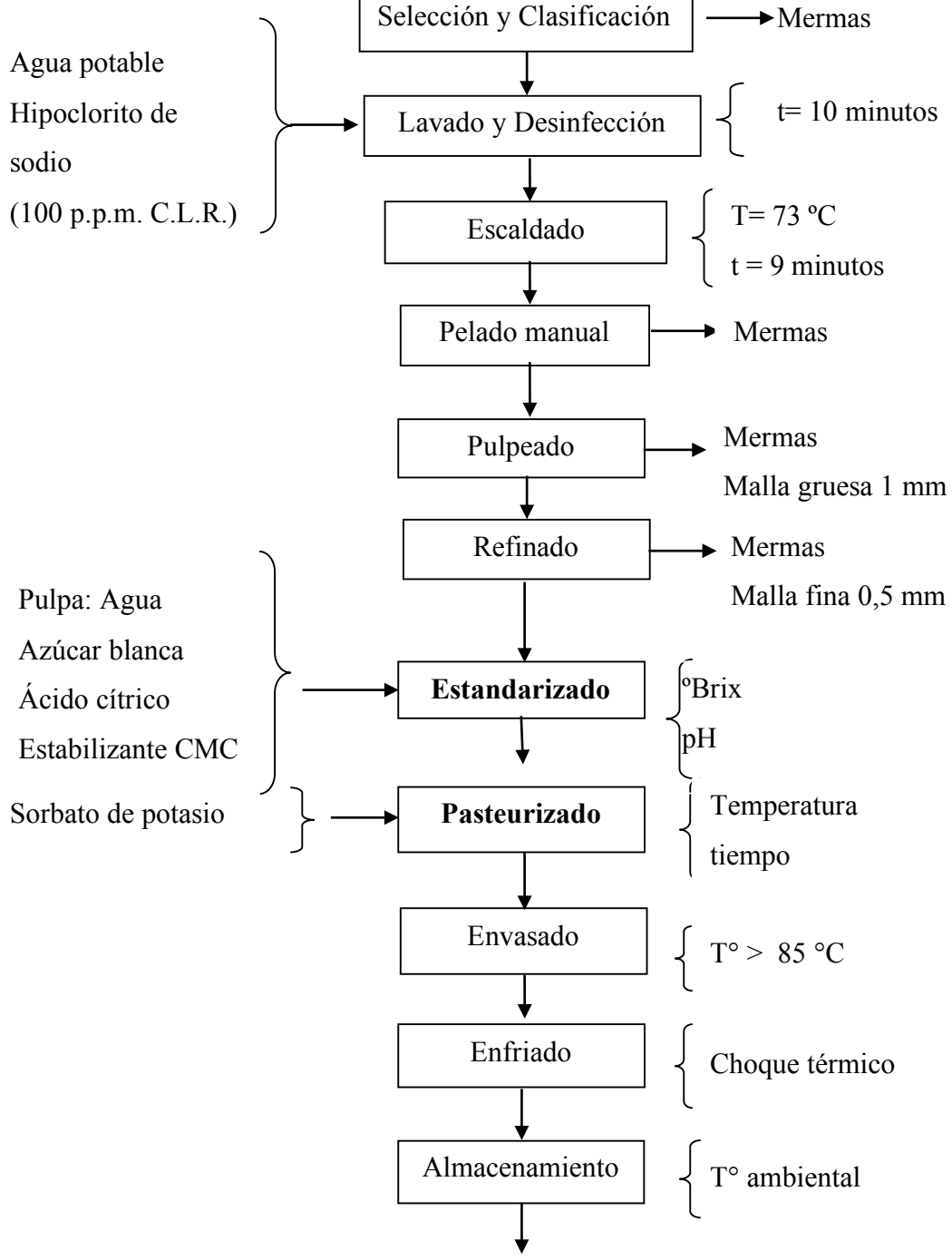

Néctar de Tomate de árbol

Figura 1. Flujo de operaciones para la obtención de néctar de tomate de árbol (Solanum betaceaum Cav.) 


\section{Análisis estadístico}

Se utilizó la metodología Taguchi ${ }^{12}$ con un arreglo ortogonal $\mathrm{L}_{9}\left(3^{4}\right)$ que comprende un diseño factorial con cuatro variables independientes, cada una con tres niveles de trabajo. Las variables dependientes fueron: contenido de ácido ascórbico, compuestos fenólicos y la capacidad antioxidante, estando combinadas mediante la función deseabilidad ${ }^{7,10}$ bajo el criterio "mayor es mejor", las cuales fueron evaluadas con el paquete estadístico Statistica versión 8 . Para las tres variables respuestas la deseabilidad global $(D)$ fue definida como la media geométrica de las deseabilidades individuales, tal como $D=(d \mathrm{AA} \times d \mathrm{CF} \times d \mathrm{CA})^{1 / 3}$. Posteriormente, aplicando la Metodología de Superficie de Respuesta, se determinaron los niveles adecuados para los factores que resultaron significativos en la etapa anterior; la función deseabilidad fue aplicada para combinar matemáticamente las tres variables respuestas y generar una función simple, mediante el análisis multirespuesta realizado por el paquete estadístico Statgraphic Centurion versión 16.1.

\section{RESULTADOS Y DISCUSIÓN}

\section{Caracterización de la materia prima}

En la tabla 2 se muestran los resultados del análisis físico-químico de la pulpa de tomate de árbol. En relación a los parámetros de $\mathrm{pH}$, acidez, sólidos solubles e índice de madurez hallados se indica que el fruto se encontraba en un estado de madurez maduro ${ }^{13}$ siendo factible su procesamiento para la elaboración de néctar, dado el desarrollo aceptable de las características sensoriales en el fruto.

Tabla 2. Composición proximal y fisicoquímica de la pulpa de tomate de árbol (Solanum betaceaum Cav.)

\begin{tabular}{lc}
\hline \multicolumn{1}{c}{ Parámetro } & Contenido \\
\hline Humedad $(\%)$ & $88,72 \pm 0,12$ \\
Proteína $(\mathrm{g} / 100 \mathrm{~g}$ de parte comestible) & $1,49 \pm 0,02$ \\
Grasa $(\mathrm{g} / 100 \mathrm{~g}$ de parte comestible) & $0,34 \pm 0,01$ \\
Fibra bruta $(\mathrm{g} / 100 \mathrm{~g}$ de parte comestible) & $1,11 \pm 0,02$ \\
Ceniza $(\mathrm{g} / 100 \mathrm{~g}$ de parte comestible) & $0,83 \pm 0,03$ \\
Carbohidratos (g/100 g de parte comestible) & $7,50 \pm 0,15$ \\
$\mathrm{pH}$ & $3,61 \pm 0,04$ \\
Sólidos solubles $\left({ }^{\circ}\right.$ Brix $)$ & $11,09 \pm 0,27$ \\
Acidez titulable $(\%$ ácido cítrico) & $1,67 \pm 0,07$ \\
Energía total (kcal/100 g de parte comestible) & $39,06 \pm 0,51$ \\
\hline \hline
\end{tabular}

\section{Análisis químico de compuestos bioactivos}

Se determinó el contenido de compuestos bioactivos en la pulpa de tomate de árbol que se muestran en la tabla 3, observándose que el contenido total de vitamina $\mathrm{C}$ se encuentra por debajo de 38,5 mg ácido ascórbico/100 $\mathrm{g}^{17}$, que incluye la forma oxidada y reducida del ácido ascórbico; los compuestos fenólicos se encuentran por debajo de $130 \mathrm{mg} \mathrm{AGE} / 100 \mathrm{~g}^{17}$ y superior a $62,71 \mathrm{mg} \mathrm{AGE} / 100 \mathrm{~g}^{16}$ la capacidad antioxidante se encuentran por encima a los 
de algunas frutas como piña con 3,4 $\mu \mathrm{mol}$ trolox/g, copuazú con 2,0 $\mu$ mol trolox/g, maracuyá con $2,7 \mu \mathrm{mol}$ trolox $/ \mathrm{g}^{11}$ tumbo serrano con $2,31 \mu \mathrm{mol}$ trolox $/ \mathrm{g}^{6}$ y por debajo de frutas como aguaymanto con $4,25 \mu \mathrm{mol}$ trolox/g, papaya de monte con $8,55 \mu \mathrm{mol}$ trolox $/ \mathrm{g}^{17}$.

Tabla 3. Análisis de los compuestos bioactivos y capacidad antioxidante de la pulpa de tomate de árbol

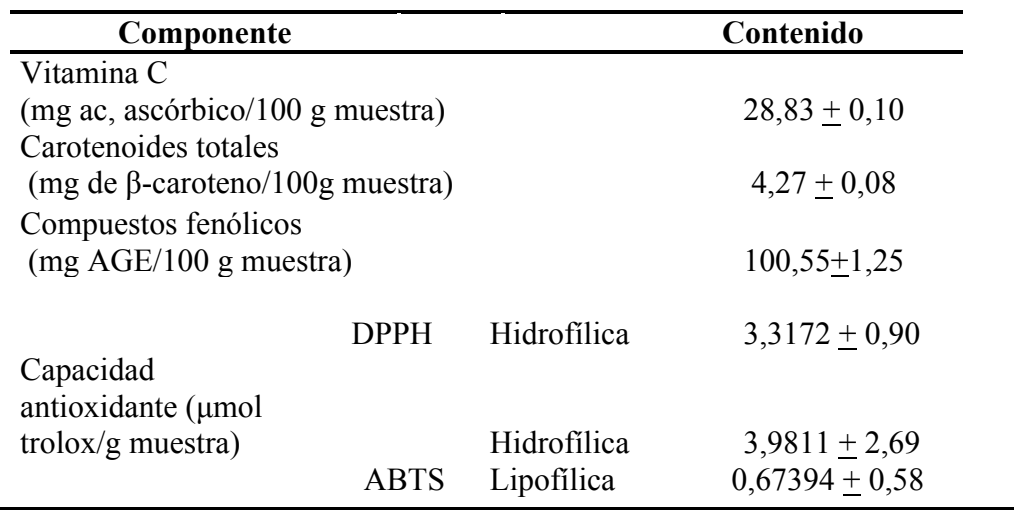

\section{Determinación de los factores $(\mathbf{p}<\mathbf{0 , 0 1})$ que influyen en la retención del ácido ascórbico, compuestos fenólicos y capacidad antioxidante en la estandarización y tratamiento térmico en la elaboración del néctar de tomate de árbol.}

En la tabla 4 se muestran los valores hallados para las variables respuesta, y la combinación de estas mediante la función deseabilidad, que permite corregir las diferencias observadas en las combinaciones de factor/nivel para cada variable. La combinación adecuada de los factores con sus respectivos niveles que garanticen una formulación óptima del néctar de tomate de árbol se obtuvo bajo el criterio "mayor es mejor", observándose en la figura 2 los valores señal/ruido (ETA) de cada factor evaluado para la función deseabilidad global $(D)$, obteniéndose una combinación adecuada factor/nivel de $\mathrm{pH}_{1}{ }^{\circ} \mathrm{Brix}_{2}$ Dilución Temperatura $_{3}$. 
Tabla 4. Resultados del contenido de ácido ascórbico, compuestos fenólicos y capacidad antioxidante del néctar de tomate de árbol

\begin{tabular}{|c|c|c|c|c|}
\hline & \multicolumn{4}{|c|}{ Variables Respuesta } \\
\hline Tratamientos & $\begin{array}{c}\text { Vitamina C } \\
\text { (mg ácido } \\
\text { ascórbico/100 g } \\
\text { muestra) } \\
\text { Y + DS }\end{array}$ & $\begin{array}{c}\text { Compuestos } \\
\text { fenólicos } \\
\text { (mg } \\
\text { AGE/100 g } \\
\text { muestra) } \\
\text { Y } \pm \text { DS }\end{array}$ & $\begin{array}{c}\text { Capacidad } \\
\text { antioxidante } \\
(\mu \mathrm{mol} / \mathrm{g} \\
\text { muestra) } \\
\text { Y } \pm \mathrm{DS}\end{array}$ & $\begin{array}{c}\text { Deseabilidad } \\
\text { global } \\
\text { promedio } \\
\text { Y }\end{array}$ \\
\hline 1 & $8,90 \pm 0,23$ & $26,29 \pm 0,09$ & $1,58 \pm 0,88$ & 0,286 \\
\hline 2 & $9,86 \pm 0,26$ & $22,28 \pm 0,81$ & $1,52 \pm 0,78$ & 0,277 \\
\hline 3 & $7,16 \pm 0,25$ & $18,18 \pm 1,00$ & $1,38 \pm 0,66$ & 0,225 \\
\hline 4 & $7,71 \pm 0,37$ & $24,87 \pm 0,72$ & $1,61 \pm 0,56$ & 0,269 \\
\hline 5 & $4,14 \pm 0,21$ & $15,89 \pm 0,58$ & $1,14 \pm 0,30$ & 0,168 \\
\hline 6 & $11,10 \pm 0,36$ & $28,30+0,64$ & $1,66 \pm 0,14$ & 0,321 \\
\hline 7 & $4,68 \pm 0,37$ & $16,87 \pm 0,34$ & $1,28 \pm 0,61$ & 0,185 \\
\hline 8 & $10,24 \pm 0,24$ & $30,35 \pm 0,76$ & $1,79 \pm 0,43$ & 0,328 \\
\hline 9 & $6,30 \pm 0,14$ & $19,93 \pm 0,69$ & $1,43 \pm 0,22$ & 0,225 \\
\hline
\end{tabular}

Los resultados fueron sometidos a un análisis de varianza (ANOVA), demostrándose que los factores con una mayor influencia significativa $(\mathrm{p} \leq 0,01)$ fueron la dilución y temperatura de tratamiento térmico con un valor $\mathrm{F}=18478,7$ y $\mathrm{F}=320,3$, respectivamente. Posteriormente, aplicando la Metodología de Superficie de Respuesta $(\mathrm{p} \leq 0,05)$, se determinaron los niveles adecuados para los factores de dilución de pulpa y temperatura de pasteurización que resultaron significativos en la etapa anterior, manteniéndose constantes el contenido de $\mathrm{pH}$ y grados brix con valores de 3,3 y 13, respectivamente; estando las tres variables respuesta combinadas matemáticamente para generar una función simple mediante la función deseabilidad. 


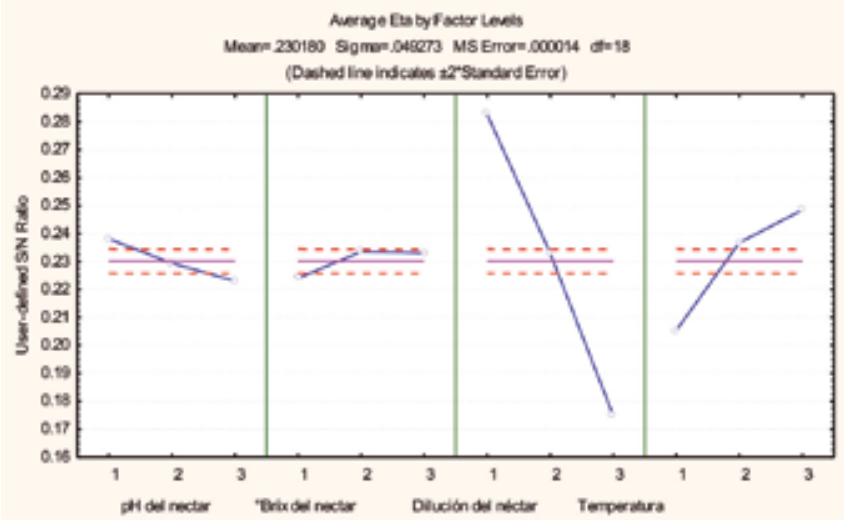

Figura 2. Valores Señal/Ruido de cada factor evaluado para una mayor retención de la función deseabilidad global en el néctar de tomate de árbol aplicando el método Taguchi $\mathrm{L}_{9}\left(3^{4}\right)$

Determinación del nivel adecuado para los factores que influyen significativamente en la retención del ácido ascórbico, compuestos fenólicos y capacidad antioxidante en la estandarización y tratamiento térmico en la elaboración del néctar de tomate de árbol.

Mediante el método de Superfice de Respueta $(\mathrm{p}<0,05)$ se determinaron los niveles adecuados de los factores en estudio que contribuyen a una mayor retención en el contenido de ácido ascórbico, compuestos fenólicos y capacidad antioxidante en la elaboración del néctar de tomate de árbol, estando las tres variables respuesta combinadas matemáticamente para generar una función simple mediante la función deseabilidad, a través del análisis multirespuesta realizado por el paquete estadístico.

En las figuras 3 y 4 se presentan las gráficas de superficie de respuesta y curvas de nivel, respectivamente; observándose los niveles óptimos de dilución y temperatura del tratamiento térmico del néctar, con una tendencia hacia los valores máximos en ambos casos. Se obtuvo una mayor retención de los compuestos bioactivos y capacidad antioxidante en el néctar de tomate de árbol con un $\mathrm{pH}$ de 3,$33 ; 13^{\circ} \mathrm{Brix}$, dilución pulpa:agua 1:2,5 y una temperatura de pasteurización de $99,5^{\circ} \mathrm{C}$ por 1 minuto.

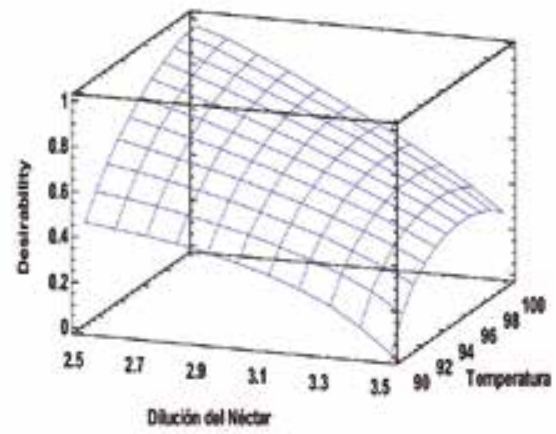

Figura 3. Gráfica de superficie de respuesta correspondiente a la función deseabilidad 


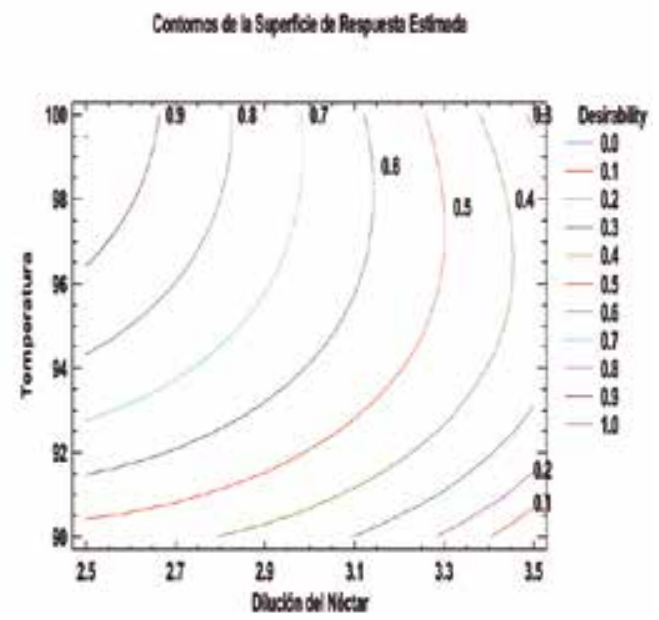

Figura 4. Curvas de nivel para la mayor retención de la función deseabilidad

\section{Caracterización del producto final}

Con los resultados obtenidos para los niveles de dilución y tratamiento térmico del néctar, y los niveles constantes de $\mathrm{pH}$ y ${ }^{\circ}$ Brix, se procedió a la elaboración del néctar de tomate de árbol cuya composición fisicoquímica se muestra en la tabla 5.

Tabla 5. Composición proximal y fisicoquímica del néctar de tomate de árbol

(Solanum betaceaum Cav.)

\begin{tabular}{lc}
\hline \multicolumn{1}{c}{ Parámetro } & Contenido \\
\hline Humedad $(\%)$ & $89,45 \pm 0,20$ \\
Proteína $(\mathrm{g} / 100 \mathrm{~g}$ de parte comestible) & $0,24 \pm 0,02$ \\
Grasa $(\mathrm{g} / 100 \mathrm{~g}$ de parte comestible) & $0,12 \pm 0,04$ \\
Fibra bruta (g/100 g de parte comestible) & $0,23 \pm 0,02$ \\
Ceniza $(\mathrm{g} / 100 \mathrm{~g}$ de parte comestible) & $0,25 \pm 0,05$ \\
Carbohidratos (g/100 g de parte comestible) & $9,71 \pm 0,17$ \\
$\mathrm{pH}$ & $3,34 \pm 0,01$ \\
Sólidos solubles ( ${ }^{\circ}$ Brix) & $13,0 \pm 0,08$ \\
Acidez titulable $(\%$ ácido cítrico) & $0,41 \pm 0,01$ \\
Energía total (kcal/100 g de parte comestible) & $40,85 \pm 0,81$ \\
\hline
\end{tabular}

El contenido de compuestos bioactivos y la capacidad antioxidante del producto obtenido se muestran en la tabla 6 , valores que determinan un porcentaje de retención de $40,42 \%$ de vitamina C, 32,78\% de compuestos fenólicos, $60,6 \%$ de carotenoides totales y 41,61 y $42,98 \%$ de capacidad antioxidante por el método DPPH y ABTS, respectivamente. Al respecto, en otras investigaciones realizadas en néctares de frutas se obtuvieron retenciones de $36,90 \%$ de vitamina $C$ para el néctar de guayaba ${ }^{14}$ retención de carotenoides totales de $61 \%$ y $70 \%$ en néctar de tuna ${ }^{8}$ y una retención del $60,48 \%$ de capacidad antioxidante hidrofílica en el néctar de tumbo ${ }^{6}$. 
Tabla 6. Análisis de los compuestos bioactivos y capacidad antioxidante del néctar de tomate de árbol

\begin{tabular}{|c|c|c|}
\hline Componente & & Contenido \\
\hline $\begin{array}{l}\text { Vitamina C } \\
\text { (mg ac. ascórbico/100 g muestra) }\end{array}$ & & $11,45+0,44$ \\
\hline $\begin{array}{l}\text { Carotenoides totales } \\
\text { (mg de } \beta \text {-caroteno } / 100 \mathrm{~g} \text { muestra) }\end{array}$ & & $1,68+0,05$ \\
\hline $\begin{array}{l}\text { Compuestos fenólicos } \\
\text { (mg AGE/100 g muestra) }\end{array}$ & & $32,96+0,64$ \\
\hline $\begin{array}{l}\text { Capacidad antioxidante } \\
(\mu \mathrm{mol} \text { trolox/g muestra })\end{array}$ & $\begin{array}{l}\text { Hidrofílica } \\
\text { Hidrofílica } \\
\text { Lipofílica }\end{array}$ & $\begin{array}{c}1,3803+2,08 \\
1,6833+0,95 \\
0,3172+0,69\end{array}$ \\
\hline
\end{tabular}

\section{CONCLUSIONES}

- La composición y características fisicoquímicas en la pulpa de tomate de árbol fueron: humedad $88,75 \%$, proteínas $1,49 \%$, grasa $0,34 \%$, fibra $1,11 \%$, cenizas $0,83 \%$, carbohidratos 7,50\%, 1,67 g de ácido cítrico/100g, 11,09 ${ }^{\circ}$ Brix y un $\mathrm{pH}$ de 3,61 con un adecuado desarrollo de las características sensoriales, favoreciendo su procesamiento para la elaboración de néctar.

- El contenido de compuestos bioactivos en la pulpa de tomate de árbol fue de: carotenoides totales con 4,27 mg $\beta$-caroteno/100g, vitamina $\mathrm{C}$ con $28,83 \mathrm{mg}$ de ácido ascórbico/100g compuestos fenólicos con 100,55 mg ácido gálico equivalente/100g y capacidad antioxidante de 3,3172 $\mu \mathrm{mol}$ trolox/g medida por el método DPPH y 4,65504 $\mu \mathrm{mol}$ trolox/g medida por el método ABTS.

- De acuerdo al método Taguchi, los factores altamente significativos sobre la función deseabilidad global, que combina el contenido de ácido ascórbico, compuestos fenólicos y capacidad antioxidante, en la elaboración del néctar de tomate de árbol fueron la dilución pulpa:agua $(1: 3)$ y la temperatura del tratamiento térmico $\left(95^{\circ} \mathrm{C}\right)$.

- Se establecieron los parámetros de elaboración del néctar de tomate de árbol que proporcionaban un mayor contenido de ácido ascórbico, compuestos fenólicos y

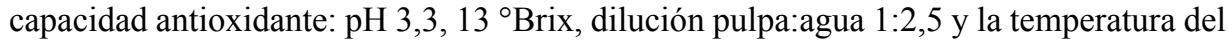
tratamiento térmico del néctar de $99,5^{\circ} \mathrm{C}$ por 1 minuto.

- La composición y características fisicoquímicas en el néctar de tomate de árbol fueron: humedad $89,45 \%$, proteínas $0,24 \%$, grasa $0,12 \%$, cenizas $0,25 \%$, carbohidratos $9,71 \%$, aporte energético 40,85 kcal/100g, 0,41 g de ácido cítrico/100g, $13{ }^{\circ}$ Brix y un $\mathrm{pH}$ de 3,34 .

- El contenido de compuestos biactivos en el néctar de tomate de árbol fue de carotenoides totales con 1,68mg $\beta$-caroteno/100g, vitamina $\mathrm{C}$ con $11,45 \mathrm{mg}$ de ácido ascórbico/100g compuestos fenólicos con 32,96mg ácido gálico equivalente/100g y capacidad antioxidante de $1,38 \mu \mathrm{mol}$ trolox/g medida por el método DPPH y 2,00 $\mu \mathrm{mol}$ trolox/g medida por el método ABTS. 


\section{AGRADECIMIENTOS}

A la Facultad de Industrias Alimentarias de la Universidad Nacional Agraria La Molina, a la Sra. Karina Capa y al Sr. Máximo Zúñiga

\section{BIBLIOGRAFÍA}

1. Ajila M, Naidu K, Bhat SG, Prasada U. Bioactive compounds and antioxidant potential of mango peel extract. Food Chem. 2005; 105: 982-988.

2. Al-Ani M, Linus O, Al-Bahri D, Al-Rahbi N. Spectrophotometric quantification of ascorbic acid contents of fruits and vegetables using the 2,4-dinitrophenylhydrazine method. JFAE; 2007; 5: 165-168.

3. AOAC (Association of Official Agricultural Chemists, US). Official Methods of Analysis, 18th edition. Washington DC: Association of Official Analytical Chemists; 2005.

4. Arnao M. Some methodological problems in the determination of antioxidant activity using chromogen radicals: A practical case. Trends Food Sci Technol. 2000; 2: 419-421

5. Brand-Williams W, Cuvelier M, Berset C. Use of a free Radical method to Evaluate Antioxidant Activity. Lebensm Wiss Tol. 1995; 28: 25-30.

6. Carpio L, Encina C. Determinación de la máxima retención de ácido ascórbico, compuestos bioactivos y capacidad antioxidante en el néctar de tumbo (Passiflora mollisima H.B.K., Bailey). Revista Ingeniería Industrial. 2009; 29: 225-247.

7. Chang M, Tsai G, Houng J. Optimization of the medium composition for the submerged cultura of Ganoderma lucidum by Taguchi array design and steepest ascent method. Enzyme Microb Technol. 2006; 38: 407-414.

8. El-Samahy SK, El-Mansy HA, Bahlol HE, El-Desouky AI, Ahmed AE. Thermal process time and sensory evaluation for canned cactus pear nectar. J Prof Assoc Cactus. 2008; 10: 85-107.

9. Howard L, Talcott S, Brenes C, Villalon B. Changes in phytochemical and antioxidant activity of selected pepper cultivars (Capsicum species) as influenced by maturity. $\mathrm{J}$ Agric Food Chem. 2000; 48 (5): 1713-1720.

10. Kim D, Rhee S. Optimization of a gas metal arc welding process using the desirability function and the genetic algorithm. Proc Inst Mech Eng, B Eng Manuf. 2004; 218 (1): $35-41$.

11. Kuskoski M, Asuero A, Troncoso A, Mancini-Filho J, Fett R. Aplicación de diversos métodos químicos para determinar la capacidad antioxidante en pulpa de frutos. Ciencia y Tecnología de Alimentos. Campinas. 2005; 25 (4): 726-732.

12. Marfil R. Una herramienta para el mejoramiento de la calidad. Tecnología de Alimentos - México. 1991; 25:5.

13. Ocampo E, Barrera E, Yepes P. Evaluación de las características fisicoquímicas del tomate de árbol (Cyphomandra betacea $\mathrm{S}$.) en tres estados de maduración. [Internet] Medellin: Facultad de Ciencias Agropecuarias, Departamento de Ingeniería Agrícola y de Alimentos. Universidad Nacional de Colombia; 2009 [citado 24 junio 2015] 
Disponible en: http://documentslide.com/documents/tomate-558b0bba21050.html

14. Ordoñez-Santos LE, Vásquez-Riascos A. Effect of processing and storage time on the vitamin $\mathrm{C}$ and lycopene contents of néctar of pink guava (Psidium guajava L.). ALAN. 2010; 6 (3): 280-284.

15. Ordoñez R, Vattuone M, Isla M. Changes in carbohydrate content and enzymes related activity during Cyphomandra betacea Sendt. fruit maturation. Postharvest Biol Technol. 2005; 35: 239-301.

16. Muñoz-Jáuregui AM, Ramos-Escudero DF, Alvarado-Ortiz C, Castañeda-Castañeda B. Evaluación de la capacidad antioxidante y contenido de compuestos fenólicos en recursos vegetales promisorios. Rev Soc Quím Perú. 2007;73 (3): 142-149.

17. Repo-Carrasco R, Encina C. Determinación de la Capacidad Antioxidante y Compuestos Bioactivos de Frutas Nativas Peruanas. Rev Soc Quím Perú. 2008; 74(2):108-124. 